\title{
Cálculo del asiento elástico tridimensional de cimentaciones de rigidez variable en terrenos con una capa rígida inclinada
}

\author{
Calculation of the three dimensional elastic settlement of foundations \\ with variable rigidity on terrains with a rigid inclined layer \\ E. Díaz ${ }^{(*)}$, R. Tomás ${ }^{(*)}$
}

RESUMEN

Las fórmulas basadas en la teoría de la elasticidad son ampliamente utilizadas para el cálculo de asientos de cimentaciones, ya que la totalidad de la normativa geotécnica recomienda su empleo. No obstante, estos métodos no cubren todas las situaciones geotécnicamente posibles ya que frecuentemente las condiciones geológicas son complejas. En este trabajo se analiza la influencia de la presencia de una capa rígida inclinada en los asientos elásticos de una cimentación superficial. Para ello se han resuelto 273 modelos tridimensionales no lineales de elementos finitos, variando los parámetros clave del problema: la inclinación y la profundidad de la capa rígida y la rigidez de la cimentación. Finalmente, se ha realizado un análisis estadístico de los resultados de los modelos y se ha propuesto una fórmula que puede ser utilizada en el cálculo de asientos por métodos elásticos, para tener en consideración la presencia de una capa rígida inclinada en profundidad.

Palabras clave: Método de elementos finitos; cimentaciones; interacción suelo-estructura; asiento; elasticidad.

\section{ABSTRACT}

The formulas based on the elasticity theory are widely used for the calculation of foundation settlements, as the geotechnical regulations recommends their use. However, these methods do not cover all possible geotechnical situations because, frequently, the geotechnical conditions are complex. In this work, the influence of an inclined rigid layer on the elastic settlements of a shallow foundation is analysed. For this purpose, 273 three-dimensional non-linear finite element models have been solved varying the key parameters of the problem: inclination and depth of the rigid layer and the rigidity of the foundation. Finally, a statistical analysis of the results of the models has been performed and a formula, which can be used for the calculation of the settlements to consider the presence of a rigid inclined layer in depth, has been proposed.

Keywords: Finite Element Method; foundations; soil-structure interaction; settlement; elasticity.

(*) Departamento de Ingeniería Civil. Escuela Politécnica Superior, Universidad de Alicante.

Persona de contacto/Corresponding author: esteban.diaz@ua.es (E. Díaz)

Cómo citar este artículo/Citation: Díaz, E., Tomás, R. (2015). Cálculo del asiento elástico tridimensional de cimentaciones de rigidez variable en terrenos con una capa rígida inclinada. Informes de la Construcción, 68(542): e144, doi: http://dx.doi.org/10.3989/ic.15.039.

Licencia / License: Salvo indicación contraria, todos los contenidos de la edición electrónica de Informes de la Construcción se distribuyen bajo una licencia de uso y distribución Creative Commons Attribution License (CC BY) Spain 3.o. 


\section{INTRODUCCIÓN}

El diseño de una cimentación superficial conlleva que ésta transmita una presión igual o inferior a la admisible del terreno y que bajo esa presión, se produzcan asientos tolerables para la estructura. Para la evaluación de estos asientos existen métodos muy sofisticados (1) que permiten simular el comportamiento del suelo de una forma mucho más precisa que los métodos basados en la teoría de la elasticidad. No obstante, la totalidad de la normativa geotécnica en vigor actualmente (2) (3) (4) recomienda el empleo de los métodos basados en la teoría de la elasticidad para la determinación del asiento de una cimentación. Probablemente, esto se deba a que llevan más de un siglo utilizándose de forma satisfactoria, ofreciendo las comparaciones existentes entre asientos medidos y calculados según esta teoría, un grado de acuerdo aceptable (5). Su fácil aplicación y la claridad y sencillez en la determinación de los parámetros intervinientes, son otras características que hacen que, en la actualidad, estas fórmulas se sigan utilizando profusamente en la mayor parte de los problemas geotécnicos, por lo que su mejora sigue siendo necesaria (6) (7) (8).

Todas las fórmulas basadas en la teoría de la elasticidad poseen un esquema común, siendo la expresión general para el cálculo de asientos elásticos de una cimentación superficial sometida una presión uniforme neta de contacto $\left(q_{\text {net }}\right)$ sobre un semiespacio elástico homogéneo e isótropo (9):

$$
s=q_{n e t} B_{e} \frac{\left(1-v^{2}\right)}{E} I
$$

donde $s$ es el asiento de la cimentación superficial, $B_{e}$ es el ancho equivalente de la cimentación, $E$ es el módulo de Young del suelo, $v$ es el coeficiente de Poisson del suelo e $I$ es el factor de influencia de desplazamientos.

El factor de influencia más utilizado es el que modifica la fórmula general en función de la forma de la cimentación (10), aunque existen otros que también pueden modificarla para cubrir aspectos tales como la rigidez relativa de la cimentación, existencia en profundidad de una capa rígida, grado de empotramiento de la cimentación, etc. Es decir, estos factores modifican la fórmula general de asientos y la adecuan a casos particulares que la fórmula general no cubre. En resumen, para determinar de la forma más exacta posible la magnitud del asiento por métodos elásticos de una cimentación superficial, es necesario el empleo de los factores de influencia, algunos de los cuales se describen en (9) (11).

Como es frecuente en la naturaleza, bajo la cimentación puede existir una capa rígida en profundidad, que suele resolverse en elasticidad mediante la analogía de capa elástica sobre base rígida. Estos casos involucran un estrato deformable limitado a cierta profundidad por una capa rígida para los niveles de cargas usuales en cimentaciones, que puede ser una roca, un suelo granular muy denso, un suelo cohesivo de consistencia muy firme, etc. En este caso existe una clara diferencia entre la distribución de tensiones y deformaciones calculadas con la teoría clásica de semiespacio elástico infinito. La influencia de esta capa es doble, por una parte, desaparece la parte de asiento correspondiente a la porción del semiespacio que ocupa, y por otra parte, alteran considerablemente la distribución de tensiones y por ende los asientos. Actualmente aunque existen soluciones teóricas (11) (12) que permiten una aproximación al problema, todas ellas contem- plan que el contacto suelo deformable-capa compresible es horizontal, además de adolecer de otra serie de deficiencias (interfaz lisa, carga flexible o cimentación perfectamente rígida, etc.) propias de cada una de las soluciones. En realidad, el contacto suelo deformable-capa rígida no suele ser horizontal, si no que suele poseer una cierta inclinación (buzamiento), esta situación puede crear giros o asientos inadmisibles en la cimentación que deben ser evaluados de una forma lo más precisa posible. Han et al. (13) realizan hasta la fecha el estudio más detallado del problema, utilizando para ello el método de las diferencias finitas, donde se pone de manifiesto la importancia de contemplar la inclinación real de la capa rígida en el cálculo de asientos, pudiéndose producir situaciones inadmisibles desde el punto de vista de los asientos, si no se tiene en consideración este hecho. No obstante, el estudio se realiza mediante la aplicación de una carga directamente en la superficie del terreno, es decir sin considerar ningún elemento transmisor de cargas (cimentación), y por lo tanto está limitado a este tipo de situaciones. En la actualidad la mayor parte de las cimentaciones gozan de una cierta rigidez, siendo perfectamente flexibles en muy pocos casos. La rigidez de una cimentación puede ser evaluada con diferentes formulaciones, quizás la más extendida sea la propuesta por Brown (14), a través del factor de flexibilidad de la cimentación $\left(K_{f}\right)$, considerándose cimentaciones perfectamente rígidas aquellas con un $K_{f}>10$, perfectamente flexibles con $K_{f}<0$,01, y de flexibilidad intermedia las que se sitúan entre ambos valores.

En consecuencia, resulta de gran interés el estudio de la influencia de la inclinación de la capa rígida en los asientos de una cimentación superficial, mediante una herramienta que permita modelizar el problema de la forma más real posible, teniendo en cuenta el carácter tridimensional del mismo, la rigidez real de la cimentación y la no linealidad debida a la interacción entre diferentes materiales como la que se produce en el contacto existente entre el terreno y la cimentación.

El objeto del presente trabajo es tratar de aprovechar la sencillez de las fórmulas de cálculo de asientos por métodos basados en la teoría de la elasticidad a través de los factores de influencia, e intentar adecuarlas a aquellos casos en los que existe la presencia de una capa rígida inclinada en profundidad. Obsérvese que en la literatura científica, no existen factores de influencia que modifiquen la fórmula general de cálculo de asientos por métodos elásticos para el caso de existencia de capa rígida inclinada en cimentaciones con cierta rigidez.

De este modo, en el presente trabajo se analizará la relación entre el asiento elástico de una cimentación superficial considerando un semiespacio elástico infinito $\left(s_{\infty}\right)$ y el asiento elástico máximo $\left(s_{\mathrm{c}}\right)$ de la misma cimentación considerando que descansa sobre una capa deformable finita bajo la que se encuentra un estrato rígido inclinado, con el fin de obtener un factor de influencia relacionado con este hecho $\left(s_{\alpha} / s_{\infty}=I_{\alpha}\right)$. Para ello, se realizarán una serie de modelos variando los parámetros que se consideran clave en la resolución del problema, utilizando la herramienta informática ANSYS que permite modelizar mediante el método de elementos finitos (que en adelante denominaremos MEF) los asientos sufridos por una estructura de cimentación.

Adicionalmente, en este artículo se propone una expresión analítica obtenida a partir del estudio estadístico de los datos, que permite determinar con la mayor exactitud el efecto de la inclinación de un sustrato rígido en el cálculo de asien- 
tos elásticos de una cimentación superficial. Esta expresión permitirá sistematizar el problema y poder resolverlo de una forma práctica y sencilla.

\section{ANÁLISIS POR ELEMENTOS FINITOS}

Con objeto de analizar la relación $\left(I_{\mathrm{q}}\right)$ entre el asiento de una cimentación superficial en un semiespacio elástico infinito $\left(s_{\infty}\right)$ con el asiento máximo de la misma cimentación en un terreno deformable bajo el cual se encuentra un sustrato rígido inclinado $\left(s_{\alpha}\right)$, se ha llevado a cabo un modelo de elementos finitos tridimensional no lineal empleando el software informático ANSYS (15) (16) (17). El proceso llevado a cabo para obtener los resultados, ha conllevado tres etapas. En la primera etapa, se ha realizado un estudio de verificación, con el fin de determinar el tamaño de malla y los límites del modelo. Posteriormente, puesto que las soluciones rigurosas elásticas disponibles únicamente contemplan el caso de capa deformable finita limitada por un sustrato rígido horizontal (12), se ha verificado el modelo de elementos finitos para el caso de capa rígida horizontal (i.e. $\alpha=0$ ) por comparación con dichas soluciones elásticas. La última etapa consiste en la determinación de los factores de influencia, utilizando el modelo verificado en los pasos anteriores.

Como modelo constitutivo del terreno y la cimentación, se ha empleado un modelo elástico lineal. Los elementos empleados han sido elementos sólidos cuadráticos de alto orden (SOLID95), elementos con 20 nodos y tres grados de libertad por nodo. Estos elementos se han empleado tanto en la cimentación como en el terreno, realizándose un mallado progresivo, con un tamaño de malla más pequeño en las inmediaciones del contacto cimentación-terreno.

Para simular el rozamiento en el contacto entre la cimentación y el suelo se han empleado elementos de interfaz o de contacto (15) (17). Concretamente, se han empleado los elementos TARGE170 y CONTA174 para simular un contacto superficiesuperficie. La rugosidad de la cimentación es simulada usando la ley de Mohr-Coulomb en el contacto entre la cimentación y el suelo, de modo que se considera una interfaz rugosa, como en la mayor parte de las cimentaciones que se construyen en la actualidad. En este caso, se ha adoptado un ángulo de rozamiento interno para dicho contacto igual a dos tercios del ángulo de rozamiento del suelo, como suele ser comúnmente aceptado para el contacto suelo-hormigón (18) y considerado en numerosas normativas técnicas. Para la resolución del problema no lineal planteado, se ha utilizado el método de Newton-Raphson que ANSYS tiene implementado. A la cimenta- ción se le carga con una fuerza axil centrada. Los parámetros base utilizados en el modelo se muestran en la Tabla 1.

Aunque el análisis se ha realizado para un coeficiente de Poisson de $1 / 3$, valor intermedio entre los que se encuentran los suelos deformables, es evidente que la distribución de tensiones en un semiespacio elástico finito es dependiente del valor del coeficiente de Poisson (11), de modo que el valor de asiento $\left(s_{\alpha}\right)$ también debe serlo. No obstante, por la propia definición realizada del factor de influencia $\left(I_{\alpha}\right)$, el término $1-v^{2}$ se encuentra tanto en el numerador como en el denominador, por lo que, el efecto del coeficiente de Poisson en el valor del factor de influencia no debería ser relevante, ya que sólo dependería del término que multiplica al factor $F_{2}$ de la formulación de Steinbrenner (12), factor con escaso peso en los resultados finales (5). No obstante, se ha realizado un estudio de sensibilidad con el valor del coeficiente de Poisson presente en suelos naturales más alejado del escogido en el presente análisis $(0,5)$. Este estudio se ha llevado a cabo con 30 modelos que se distribuyen cubriendo las diferentes situaciones estudiadas, en los que se han comparado los factores de influencia $\left(I_{\alpha}\right)$ obtenidos con el valor de coeficiente de Poisson utilizado en el presente trabajo $(0,33)$ y el extremo $(0,5)$. Los resultados indican un error medio del $0,47 \pm 0,35 \%$. Partiendo del hecho de que se ha analizado el caso más desfavorable y con los valores extremos, se puede concluir que el efecto del coeficiente de Poisson no es determinante, hecho que por otra parte era de esperar a la vista de cómo se ha definido el factor de influencia en el presente trabajo.

Obsérvese que no ha sido necesario considerar variables el resto de parámetros que intervienen en el cálculo de asientos $\left(B, q_{n e t} \mathrm{y} E\right)$, dado que el cálculo del factor de influencia es independiente de ellos, al encontrarse tanto en el numerador como en el denominador.

El canto de la cimentación se determina en función de los diferentes valores del factor de flexibilidad de la cimentación $\left(K_{f}\right)$, de modo que se variará con objeto de obtener cimentaciones de diferente flexibilidad. En todos los casos existe un elemento transmisor de cargas al suelo definido por un material con un determinado módulo de Young $\left(E_{f}\right)$ muy superior al del suelo infrayacente.

A una profundidad $z$, bajo el punto medio de la cimentación se ha situado una capa rígida en profundidad, con los parámetros mostrados en la Tabla $1\left(E_{b}\right.$ y $\left.v_{b}\right)$, estos parámetros suponen para el rango de presiones ordinario en cimentaciones superficiales, que el material puede considerarse indeformable.

Tabla 1. Parámetros base adoptados en el modelo.

\begin{tabular}{|l|c|c|c|}
\hline Parámetro & Símbolo & Unidades & Valor adoptado \\
\hline Axil aplicado & $N$ & $\mathrm{kN}$ & 800 \\
\hline Ancho de la cimentación & $B$ & $\mathrm{~m}$ & 2 \\
\hline Largo de la cimentación & $L$ & $\mathrm{~m}$ & 2 \\
\hline Módulo de Young de la cimentación & $E_{f}$ & $\mathrm{kPa}$ & $3 \cdot 10^{7}$ \\
\hline Coeficiente de Poisson de la cimentación & $v_{f}$ & - & 0,20 \\
\hline Módulo de Young del terreno suprayacente & $E$ & $\mathrm{kPa}$ & $2 \cdot 10^{4}$ \\
\hline Coeficiente de Poisson del terreno suprayacente & $v$ & - & $1 / 3$ \\
\hline Módulo de Young del terreno rígido & $E_{b}$ & $\mathrm{kPa}$ & $4 \cdot 7 \cdot 10^{7}$ \\
\hline Coeficiente de Poisson del terreno rígido & $v_{b}$ & - & 0,19 \\
\hline
\end{tabular}


La condición simétrica del problema ha conllevado la confección de únicamente un medio del modelo aplicando condiciones de contorno de simetría en el plano medio. En la base del modelo se han fijado los desplazamientos verticales y en los laterales (donde no existe simetría) se han fijado los desplazamientos horizontales (Figura 1). Los resultados de los análisis previos para determinar el tamaño de malla apropiado y los límites del modelo (base y laterales), han determinado que se adoptará un mallado de o,1 m en la cimentación y en el suelo en las inmediaciones de la cimentación, que progresivamente ha ido decreciendo a $0,5 \mathrm{~m}$ en los bordes del modelo. El límite inferior ha sido fijado en 22 veces el ancho de la cimentación y los límites laterales en 14 veces el ancho de la misma. Con estas dimensiones se procede a comparar los resultados obtenidos del MEF con la solución rigurosa propuesta por Steinbrenner (12) para la existencia de una capa horizontal rígida en profundidad, obteniéndose un error medio del 3,75 $\pm 1,17 \%$, verificándose así la validez del modelo y el tamaño de malla utilizado.

En la Figura 1 se muestra un esquema del modelo adoptado para el caso de presencia de una capa rígida a una profundidad $z$ por debajo de la base de la cimentación.

\section{OBTENCIÓN DEL FACTOR DE INFLUENCIA}

Una vez realizado el estudio de verificación del modelo, se procede a resolver el mismo para los diferentes supuestos, los pasos que se han seguido se detallan a continuación:

1. ${ }^{\circ}$ Cálculo del asiento elástico máximo $\left(s_{\alpha}\right)$ de una cimentación de una determinada rigidez $\left(K_{f}\right)$ apoyada en una capa deformable finita con unos parámetros elásticos fijos $(E \mathrm{y} v$, ver Tabla 1) bajo la que se encuentra a una profundidad $z$, una capa rígida inclinada un ángulo a respecto a la horizontal.

2. ${ }^{\circ}$ Para la misma cimentación se calcula el asiento elástico $\left(s_{\infty}\right)$ considerando la cimentación perfectamente flexible y suponiendo que la misma se apoya en un semiespacio elástico infinito con los mismos parámetros elásticos del terreno suprayacente $(E \mathrm{y} v)$ utilizados anteriormente.

$3 .^{\circ} \mathrm{El}$ proceso se repite para diferentes valores de la profundidad de la capa compresible ( $z$ ), ángulo de inclinación de la capa rígida ( $\alpha$ ) y rigidez de la cimentación $\left(K_{f}\right)$.

$4 .^{\circ}$ La relación $s_{\alpha} / s_{\infty}$ es el factor de influencia $I_{\alpha}$.

$5 .^{\circ}$ Adicionalmente para todos los casos estudiados se ha obtenido el perfil de asientos a lo largo del plano medio de la cimentación.

De este modo, simplemente calculando el asiento de una cimentación en un semiespacio elástico infinito (9) (10) y aplicando el factor de influencia propuesto en el presente trabajo, puede obtenerse de una forma sencilla, rápida y precisa el asiento de una cimentación apoyada en un estrato deformable de espesor finito bajo el que se sitúa una capa rígida inclinada.

En total se han resuelto 273 modelos, variando gradualmente los parámetros determinantes y anteriormente comentados en el cálculo de asientos.

\section{PRESENTACIÓN DE LOS RESULTADOS DE LA INVESTIGACIÓN}

De acuerdo a los métodos presentados en el apartado anterior se han obtenido 273 valores del factor de influencia $I_{a}$.
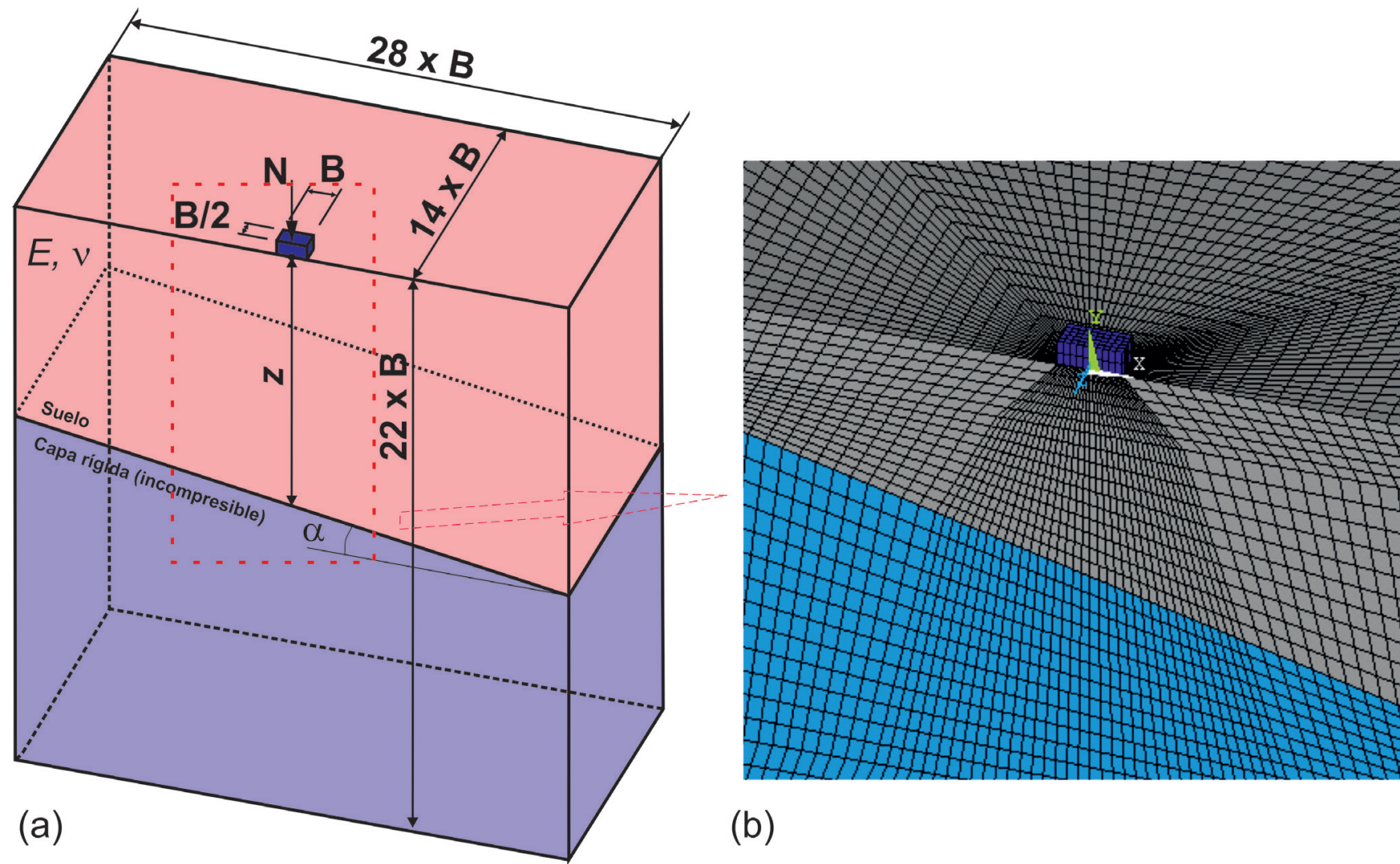

Figura 1. a) Esquema del modelo de elementos finitos adoptado. b) Porción ampliada del modelo con detalle de la malla utilizada (caso particular $\alpha=30$ ). 
Los modelos se agrupan según diferentes relaciones entre la profundidad de la capa rígida respecto al ancho de la cimentación $(z / B)$, en concreto se han resuelto los casos $0,25,0,5$, $0,75,1,1,5,2,3,5$ y 10, medidos siempre desde el punto central de la cimentación hasta el techo de la capa rígida.

Todos estos casos se resuelven para 5 diferentes valores de inclinación de la capa rígida $\left(0^{\circ}, 15^{\circ}, 30^{\circ}, 45^{\circ}\right.$ y $\left.60^{\circ}\right)$. Estas inclinaciones se miden siempre paralelas a la dirección del ancho de la cimentación. Existen ciertos casos que por cuestiones geométricas no se han resuelto, ya que debido a la relación entre $z / B$ y $\alpha$, la superficie de contacto que define el techo de la capa rígida intersecta la cimentación.

Finalmente, todos estos casos se resuelven para diferentes valores de rigidez relativa definida a través del factor de flexibilidad (14) de la cimentación (100, 30, 10, 1, 0,1, 0,01, 0,001) que cubren todo el espectro de rigidez relativa que presentan la mayor parte de las cimentaciones que se construyen en la actualidad.

En la Figura 2, se observa la variación del factor de influencia $\left(I_{a}\right)$ respecto a la relación $z / B$ para todos los casos de inclinación de la capa rígida considerados y para cuatro valores del factor de flexibilidad estudiados (100, 10, 0,1, 0,001), los casos no representados $(30,1,0,01)$ muestran una tendencia intermedia entre estos. La tendencia general es un aumento del factor de influencia a medida que disminuye la rigidez de la cimentación para una inclinación fija del sustrato rígido, situándose en valores muy cercanos a 1 para el caso de la cimentación con menor rigidez y con la relación $z / B$ más alta analizada. Del mismo modo, para un factor de flexibilidad fijo, se produce un aumento del factor de influencia a medida que aumenta la inclinación del sustrato rígido.

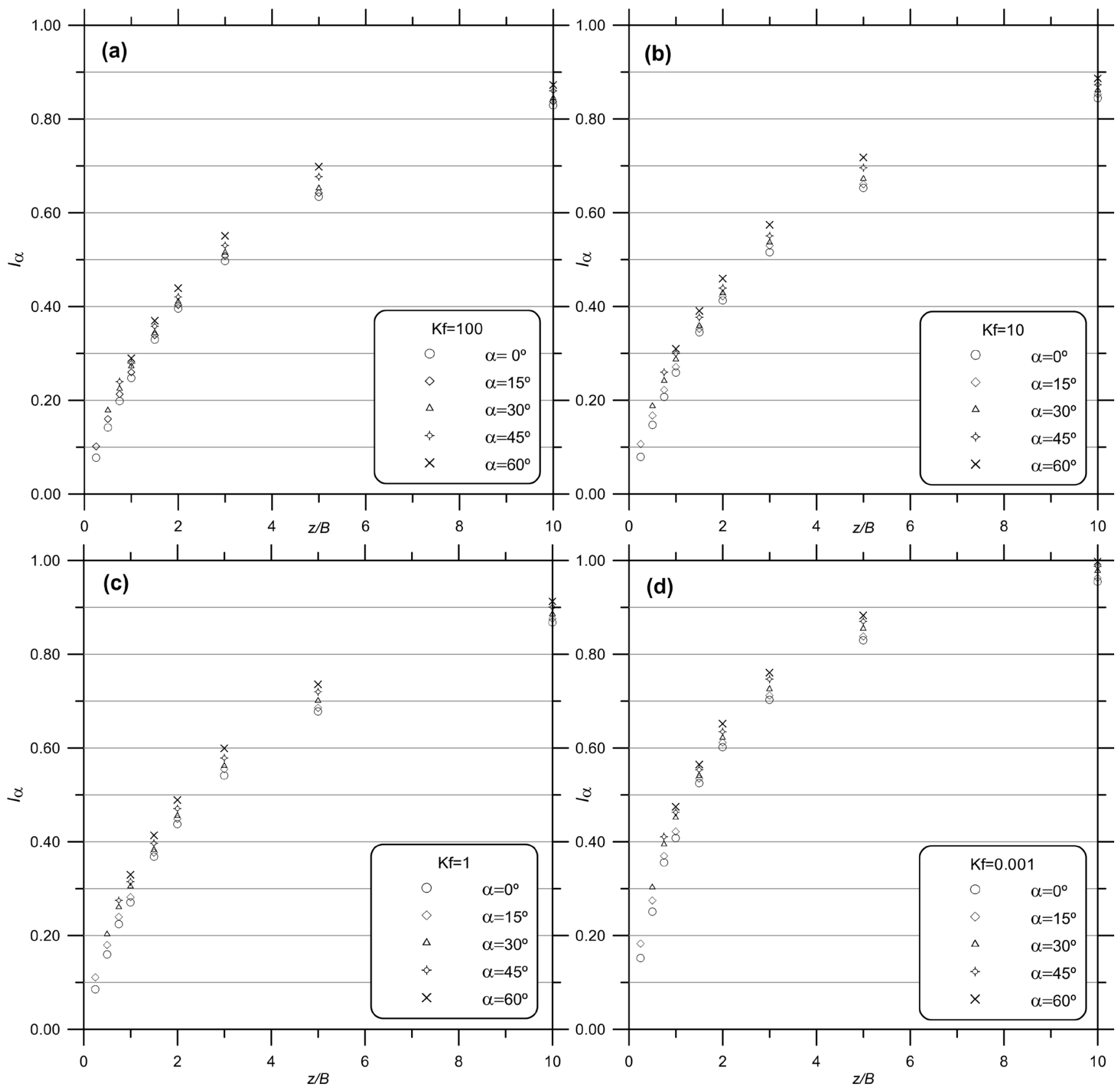

Figura. 2. Variación del factor de influencia para el caso de inclinación del sustrato rígido, para las diferentes relaciones z/B y ángulos de inclinación estudiados. La figura incluye los casos de rigidez relativa extremos y frontera entre comportamientos, a) $K_{f}=100$, b) $K_{f}=10$, c) $\left.K_{f}=1 \mathrm{y} \mathrm{d}\right) K_{f}=0,001$. 
Tabla 2. Valores del factor de influencia $\left(I_{\alpha}\right)$ obtenidos para el caso particular $\left.K_{f}=100 .{ }^{*}\right)$ Casos que no tienen solución por limitaciones geométricas (el techo de la capa rígida intersecta el plano de cimentación por la propia cimentación).

\begin{tabular}{|c|c|c|c|c|c|c|}
\hline \multicolumn{2}{|c|}{ Caso $\rightarrow$} & \multirow{2}{*}{$\begin{array}{c}\begin{array}{c}\text { Capa } \\
\text { horizontal }\end{array} \\
\mathbf{o} \\
\end{array}$} & \multicolumn{4}{|c|}{ Capa inclinada } \\
\hline $\mathbf{z} / \boldsymbol{B} \downarrow$ & $\alpha(\mathbf{0}) \rightarrow$ & & 15 & 30 & 45 & 60 \\
\hline \multicolumn{2}{|c|}{0,25} & 0,0778 & 0,1021 & * & * & * \\
\hline \multicolumn{2}{|c|}{$\mathbf{0 , 5}$} & 0,1423 & 0,1605 & 0,1790 & * & $*$ \\
\hline \multicolumn{2}{|c|}{0,75} & 0,1985 & 0,2135 & 0,2250 & 0,2403 & $*$ \\
\hline \multicolumn{2}{|c|}{1} & 0,2478 & 0,2602 & 0,2733 & 0,2798 & 0,2900 \\
\hline \multicolumn{2}{|c|}{$\mathbf{1 , 5}$} & 0,3299 & 0,3392 & 0,3440 & 0,3580 & 0,3702 \\
\hline \multicolumn{2}{|c|}{2} & 0,3958 & 0,4034 & 0,4109 & 0,4209 & 0,4395 \\
\hline \multicolumn{2}{|c|}{3} & 0,4971 & 0,5086 & 0,5156 & 0,5306 & 0,5510 \\
\hline \multicolumn{2}{|c|}{5} & 0,6346 & 0,6426 & 0,6526 & 0,6770 & 0,6984 \\
\hline \multicolumn{2}{|c|}{10} & 0,8294 & 0,8374 & 0,8445 & 0,8604 & 0,8733 \\
\hline
\end{tabular}

En la Tabla 2, se muestran los valores numéricos del factor de influencia $\left(I_{\alpha}\right)$ para el caso particular $K_{f}=100$ y para todas las relaciones $z / B$ estudiadas y casos de inclinación ( $\alpha$ ) del sustrato rígido considerados, con objeto de poder comparar la variación entre la consideración del estrato rígido horizontal y con las distintas inclinaciones estudiadas.

Como se ha mencionado en secciones anteriores, para la obtención del factor de influencia siempre se ha tenido en cuenta el asiento máximo de la cimentación, que dependiendo de la inclinación del sustrato rígido y de la rigidez relativa de la cimentación, puede ir variando de posición. A partir del estudio de todos los perfiles de asientos obtenidos, se han podido establecer dos tipos de comportamientos de las cimentaciones consideradas, un comportamiento asimilable al de una cimentación rígida, para factores de flexibilidad entre $10 \mathrm{y}$ 100; y un comportamiento asimilable al de una cimentación flexible, con factores de flexibilidad entre 1 y o,oo1. A continuación, se detallan las características más significativas de ambos comportamientos.
Como se ha comentado, para factores de flexibilidad $\left(K_{f}\right)$ entre 100 y 10 , se observa un comportamiento asimilable al de una cimentación rígida, obteniéndose perfiles de asientos predominantemente lineales y que varían según la inclinación del sustrato. Para el caso horizontal $(\alpha=0)$ se obtiene el valor máximo en el centro y los mínimos en los bordes, con pequeñas diferencias entre ambos. Una vez que el sustrato se inclina, se produce un perfil de asientos lineal con un mínimo en el borde de la cimentación situado en la zona con menor espesor de estrato compresible y un máximo en el borde opuesto, acentuándose la diferencia a medida que aumenta la inclinación. Del mismo modo, a medida que aumenta la relación $z / B$, este perfil se ve modificado paulatinamente para ir pareciéndose más al perfil de asientos del caso de capa rígida horizontal, aunque siempre manteniendo el máximo en la posición del borde situado en la zona con mayor espesor de terreno deformable y reduciéndose la diferencia entre los valores de asiento máximo y mínimo. Todos estos aspectos se representan en la Figura 3, mostrándose los casos límite del factor de flexibilidad en los que la cimentación posee un com-

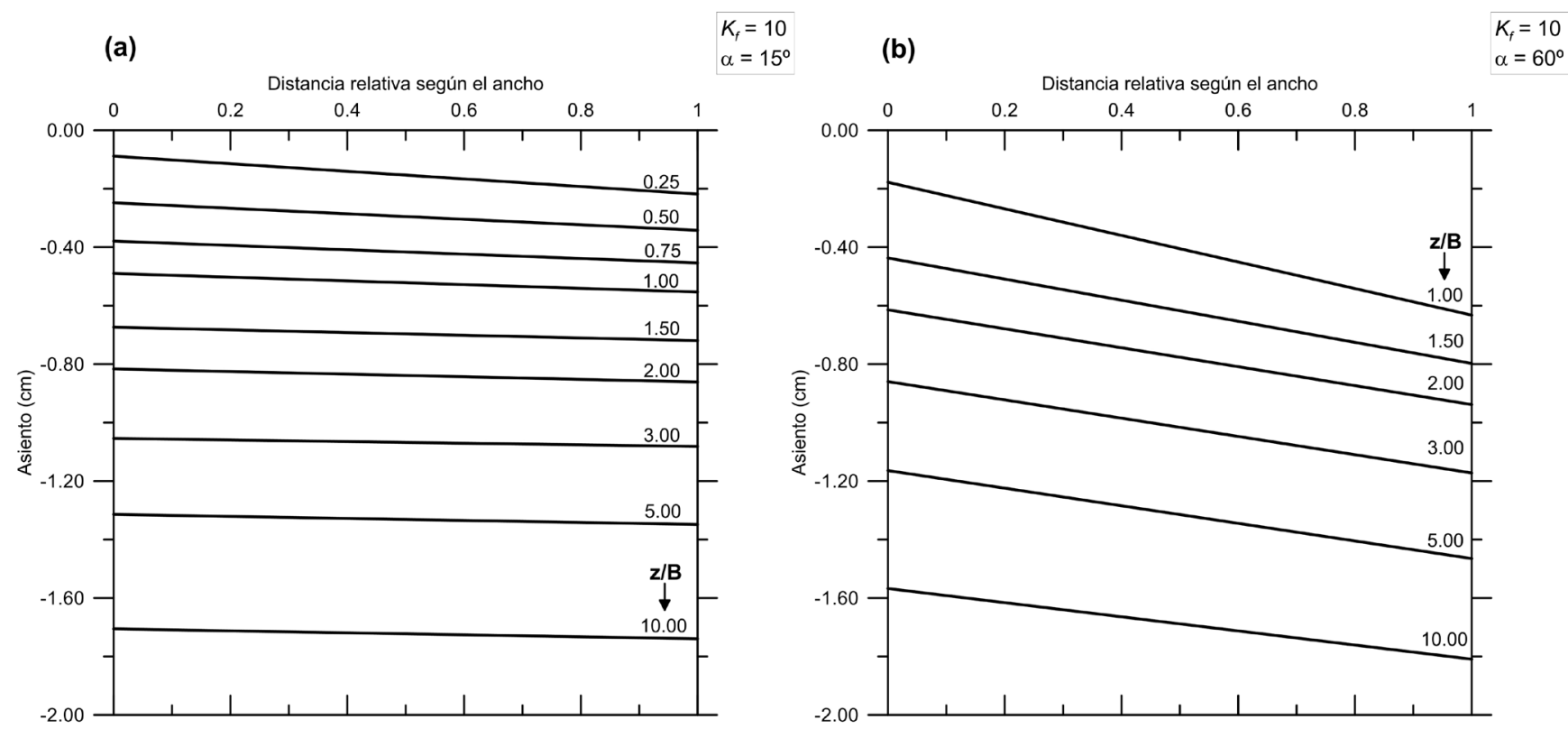

Figura 3. Perfil de asientos para el caso de cimentación cuyo comportamiento puede considerarse rígido, con menor factor de flexibilidad $\left(K_{f}=10\right)$ para todas las relaciones $z / B$ estudiadas. Se presentan los casos extremos de inclinación del sustrato rígido, a) $\alpha=15^{\circ}$ y b) $\alpha=60^{\circ}$. Datos tomados en el plano medio de la cimentación. 
portamiento asimilable al de una cimentación rígida $(100 \geq$ $K_{f} \geq 10$ ) y para los casos extremos de inclinación del sustrato rígido $\left(\alpha=15^{\circ}\right.$ y $\left.60^{\circ}\right)$.

A partir de un valor del factor de flexibilidad igual a 1, la cimentación posee un comportamiento que ya no puede considerarse rígido, produciéndose un cambio en la forma de los perfiles de asientos, para el caso de sustrato horizontal el perfil sigue manteniendo un mínimo en las esquinas y un máximo en el centro, aunque con diferencias más acentuadas y careciendo del perfil lineal anteriormente expuesto. A medida que aumenta la inclinación de la capa rígida, se producen diferencias superiores entre los valores mínimo y máximo; y además el valor máximo ya no se sitúa en el extremo de la cimentación en el que existe el mayor espesor de terreno deformable, si no que se sitúa en una posición intermedia entre el punto medio y dicho extremo, aunque más cerca del extremo para inclinaciones de sustrato altas y/o relaciones $z / B$ bajas, para ir acercándose al punto medio a medida que disminuye la inclinación del sustrato o aumenta la relación $z / B$. Todas estas tendencias pueden contemplarse en la Figura 4.

\section{AJUSTE DE DATOS Y FORMULACIÓN PROPUESTA PARA EL FACTOR DE INFLUENCIA}

De acuerdo a las consideraciones anteriores y teniendo en cuenta las variables que determinan el valor del factor de influencia $\left(K_{f}, \alpha, z / B\right)$, en el presente apartado se exponen los resultados del análisis estadístico realizado con los datos obtenidos experimentalmente. El análisis estadístico ha consistido en un ajuste de un hiperplano a los datos derivados de los modelos resueltos mediante una regresión múltiple no

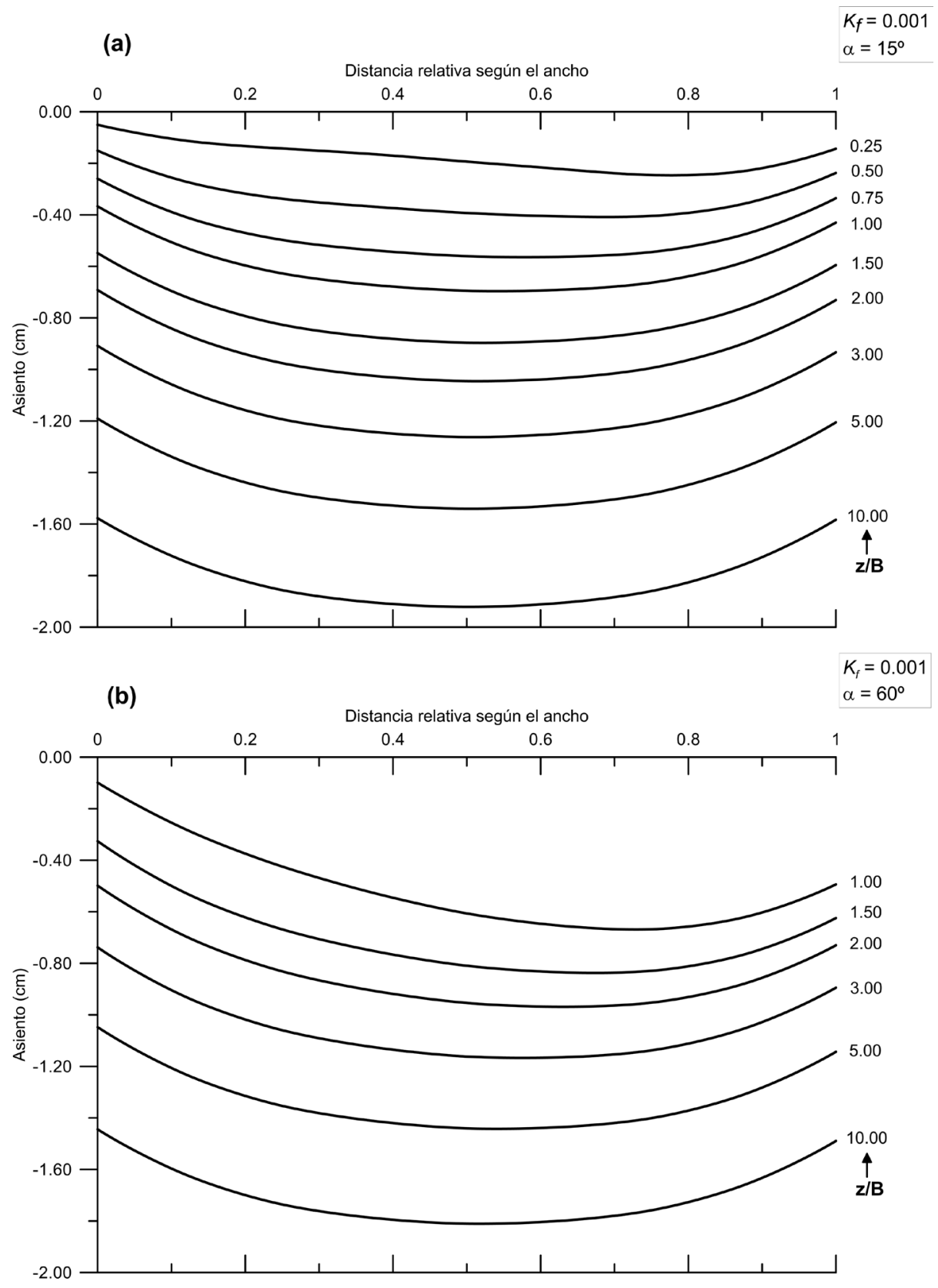

Figura 4. Perfil de asientos para el caso de cimentación cuyo comportamiento puede considerarse flexible, con menor factor de flexibilidad $\left(K_{f}=0.001\right)$ para todas las relaciones $z / B$ estudiadas. Se presentan los casos extremos de inclinación del sustrato rígido, a) $\alpha=15^{\circ} \mathrm{y}$ b) $\alpha=60^{\circ}$. Datos tomados en el plano medio de la cimentación. 
lineal y una comprobación de su grado de exactitud mediante el coeficiente de determinación $\left(R^{2}\right)$ y el error relativo medio.

Para realizar el ajuste de los datos experimentales, se ha utilizado el programa POLY-MODEL (19) que permite la generación de familias de modelos matemáticos con parámetros no lineales basados en los datos experimentales de las variables intervinientes, empleando el algoritmo de Gauss-Newton.

La fórmula obtenida se presenta en la ecuación [2], Obteniéndose un coeficiente de determinación $\left(R^{2}\right)$ de 0,991 y un error relativo medio del 2,41 $\pm 2,18 \%$. En la Figura 5 se representan los valores obtenidos mediante el MEF del factor de influencia $\left(I_{\alpha}\right)$ y los estimados mediante la ecuación [2], además se han incorporado los límites del error para el intervalo $\pm 5 \%$.

$$
\begin{aligned}
& I_{E P}=0,1261 \cdot e^{-\left(8,7510 \cdot K_{f}+0,0949\right)}+\left(\frac{1}{1529} \cdot \alpha+0,7690\right)^{2}- \\
& -1,1715 \cdot e^{-\left(0,4892 \cdot \frac{Z}{B}+0,7061\right)}-0,0002 \cdot K_{f}+\frac{Z}{B} \cdot 0,0249
\end{aligned}
$$

Los valores límite para la aplicación de la fórmula son:

$$
100 \geq K_{f} \geq 0,001,10 \geq z / B \geq 0,25 \text { y } \alpha \leq 60^{\circ}
$$

Por lo tanto, la ecuación [2] permite calcular el factor de influencia $\left(I_{\alpha}\right)$ que considera el asiento elástico máximo de una cimentación de rigidez $K_{f}$, que se sitúa sobre una capa deformable finita de espesor $z$, bajo la que se encuentra un estrato rígido inclinado un ángulo $\alpha$. Este factor de influencia modifica la fórmula general de cálculo de asientos por métodos elásticos [ecuación 1], adecuándola al caso estudiado en el presente trabajo. La expresión tendría la forma:

$$
s=q_{n e t} B_{e} \frac{\left(1-v^{2}\right)}{E} I_{\alpha}
$$

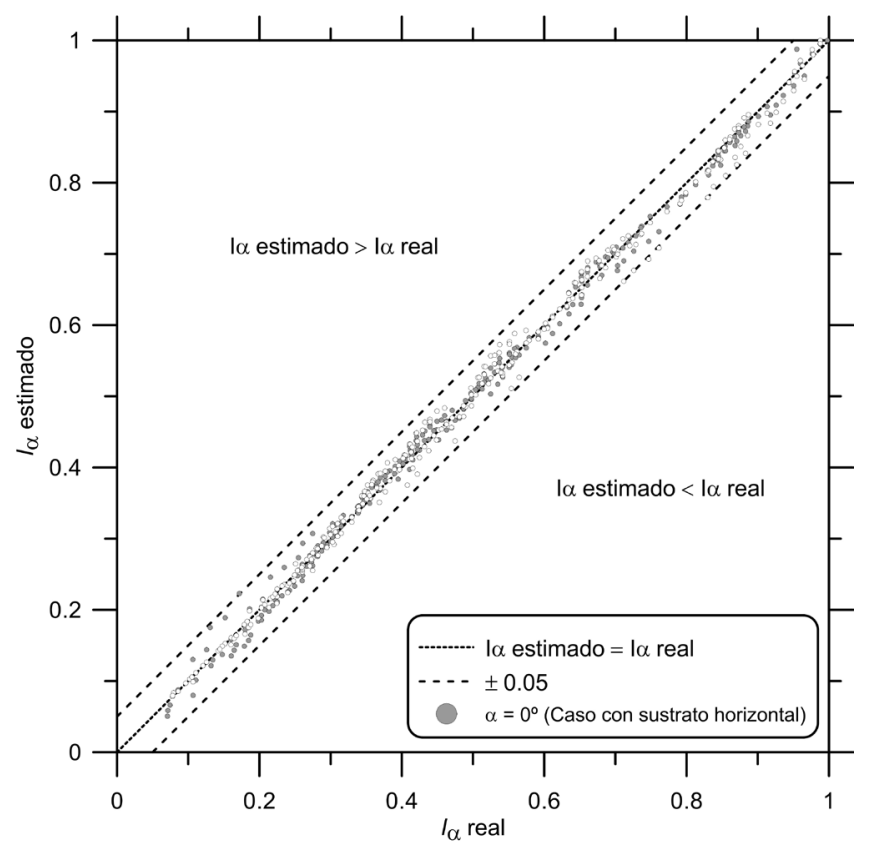

Figura 5. Comparación entre los valores del factor de influencia $\left(I_{\mathrm{a}}\right)$ reales obtenidos mediante el MEF y los estimados mediante la ecuación [2]. Se han incluido mediante puntos grises los casos correspondientes al caso de sustrato rígido horizontal $(\alpha=0)$.

\section{CONCLUSIONES}

Se ha llevado a cabo un análisis tridimensional no lineal mediante el MEF con el objeto de analizar la relación entre el asiento de una cimentación superficial en un semiespacio elástico infinito, con el asiento máximo de la misma cimentación en un terreno deformable bajo el cual se encuentra un sustrato rígido inclinado $\left(s_{\alpha} / s_{\infty}=I_{\alpha}\right)$, para ello se han realizado un total de 273 modelos. Las conclusiones más relevantes extraídas de este estudio son:

1. Se ha realizado un modelo mediante el MEF resultado de un estudio previo de verificación, cuyos resultados ponen de manifiesto la validez del modelo empleado.

2. El estudio se ha efectuado bajo unas condiciones similares a las de la mayor parte de las cimentaciones superficiales construidas en la actualidad (cimentación de rigidez variable, de hormigón armado, interfaz rugosa, etc.) y estudiando un número considerable de casos. Este estudio tridimensional comparativo entre el asiento de una cimentación superficial en un semiespacio elástico infinito y el asiento máximo de la misma cimentación en un terreno bajo el cual se encuentra un sustrato inclinado, analizando un número tan elevado de casos, no existe en la bibliografía científica.

3. El estudio revela que la inclinación del sustrato rígido, en muchos casos, afecta de forma determinante al asiento de una cimentación superficial. De modo que el conocer con precisión este valor, es importante a la hora de garantizar que los asientos de la cimentación sean admisibles.

4. El comportamiento de las cimentaciones superficiales estudiadas, es muy diferente según su factor de flexibilidad, pudiéndose distinguir un primer grupo $\left(100 \geq K_{f} \geq 10\right)$ en el que el comportamiento se asimila al de una cimentación rígida, obteniéndose los valores de asiento máximo en el extremo de la cimentación donde existe mayor espesor de terreno compresible. En el segundo grupo ( $1 \geq K_{f} \geq 0$,001), el comportamiento ya no es de una cimentación rígida, si no que el valor máximo del asiento se suele localizar entre el punto central de la cimentación y el borde, en posiciones más cercanas al extremo para inclinaciones de sustrato altas y/o relaciones $z / B$ bajas, para ir paulatinamente aproximándose al punto central según disminuye la inclinación del sustrato o aumenta la relación $z / B$.

5. Del análisis estadístico de los datos, una nueva fórmula que permite sistematizar el problema estudiado para su uso práctico, ha sido presentada para la obtención del factor de influencia $\left(I_{\alpha}\right)$. La formulación posee un coeficiente de determinación $\left(R^{2}\right)$ de 0,991 , y un error relativo medio del 2,41 $\pm 2,18 \%$. Esta formulación relaciona la rigidez de una cimentación superficial a través del factor flexibilidad, la relación $z / B$ y el ángulo de inclinación del sustrato rígido con el factor de influencia $\left(I_{\alpha}\right)$.

6. Su fácil aplicación, su grado de exactitud y su bajo error, la convierten en una herramienta interesante para el cálculo del asiento máximo de una cimentación superficial en el caso de que bajo la misma exista una capa rígida inclinada, cuando no se disponen de herramientas más sofisticadas (software MEF), pudiéndose implementar en las fórmulas de cálculo de asientos basadas en la teoría de la elasticidad incluidas en la normativa geotécnica actual. 


\section{AGRADECIMIENTOS}

Los autores agradecen al grupo de investigación «Modelización Matemática de Sistemas» de la Universidad de Alicante, la ayu- da prestada en el ajuste de los datos experimentales y especialmente al Dr. José Antonio Reyes. El trabajo se ha desarrollado en el marco del proyecto del Ministerio de Economía y Competitividad de España y los Fondos Feder TEC2011-28201-Co2-02.

\section{REFERENCIAS}

(1) Ti, K. S., Huat, B. B., Noorzaei, J., Jaafar, M. S., \& Sew, G. S. (2009). A review of basic soil constitutive models for geotechnical application. Electronic Journal of Geotechnical Engineering, 14: 1-18.

(2) AASHTO. (2010). AASHTO LRFD Bridge design specifications, 5th ed. Washington D.C.: American Association of State Highway and Transportation Officials.

(3) CEN. (2004). Eurocode 7: Geotechnical design. ENV 1997:2004, CEN, Brussels.

(4) CTE. (2006). Código Técnico de la Edificación. Documento básico de seguridad estructural-cimientos. Ministerio de Fomento de España.

(5) Bowles, J. (1987). Elastic foundation settlements on sand deposits. Journal of Geotechnical Engineering, 113(8): 84686o. doi: http://dx.doi.org/10.1061/(ASCE)0733-9410(1987)113:8(846)

(6) Shin, E., \& Das, B. (2011). Developments in elastic settlement estimation procedures for shallow foundations on granular soil. KSCE Journal of Civil Engineering, 15(1): 77-89. doi: http://dx.doi.org/10.1007/s12205-011-1028-9.

(7) Naseri, M., \& Hosseininia, E. S. (2015). Elastic settlement of ring foundations. Soils and Foundations, 55(2): $284-295$. doi: http://dx.doi.org/10.1016/j.sandf.2015.02.005.

(8) Maheshwari, P. (2015). Settlement of shallow footings on layered soil: state-of-the-art. International Journal of Geotechnical Engineering, 9(1): 42-48. doi: http://dx.doi.org/10.1179/1939787914Y.0000000065.

(9) Mayne, P., \& Poulos, H. (1999). Approximate displacement influence factors for elastic shallow foundations. Journal of Geotechnical and Geoenvironmental Engineering, 125(6): 453-46o. doi: http://dx.doi.org/10.1061/(ASCE)10900241(1999)125:6(453).

(10) Schleicher, F. (1926). Zur theorie des baugrundes. Bauingenieur Band, 7: 931-935, 949-952.

(11) Milovic, D. (1992). Stresses and displacements for shallow foundations. Elsevier Science.

(12) Steinbrenner, W. (1934). Tafeln zur setzungsberechnung. Die Strasse, 1: 121-124.

(13) Han, J., Huang, J., \& Parsons, R. L. (2007). Influence of bedrock inclination on elastic settlements of flexible shallow strip foundations. Computers and Geotechnics, 34(1): 53-56. doi: http://dx.doi.org/10.1016/j.compgeo.2006.09.004.

(14) Brown, P. T. (1969). Numerical analyses of uniformly loaded circular rafts on deep elastic foundations. Géotechnique, 19: 399-404.

(15) Ansys. (2007a). Elements Reference. Ansys Release 11.o Documentation. Ansys Inc.

(16) Ansys. (2007b). Theory Reference. Ansys Release 11.o Documentation. Ansys Inc.

(17) Ansys. (2007c). Contact Technology Guide. Ansys Release 11.o Documentation. Ansys Inc.

(18) Potyondy, J. G. (1961). Skin friction between various soils and construction materials. Géotechnique, 11: 339-353.

(19) Verdú, F., \& Villacampa, Y. (2008). A computational algorithm for the multiple generation of nonlinear mathematical models and stability study. Advances in Engineering Software, 39(5): 430-437. doi: http://dx.doi.org/10.1016/j.advengsoft.2007.03.004. 